\title{
Full endoscopic resection of a lumbar osteoblastoma: technical note
}

\author{
William C. Newman, MD, ${ }^{1}$ Max Vaynrub, MD, ${ }^{2}$ Mark H. Bilsky, MD, ${ }^{1,3}$ llya Laufer, MD, MS, ${ }^{1,3}$ and \\ Ori Barzilai, MD'
} Departments of ${ }^{1}$ Neurological Surgery and ${ }^{2}$ Orthopedic Oncology, Memorial Sloan Kettering Cancer Center; and ${ }^{3}$ Department of
Neurological Surgery, Weill Cornell Medical College, NewYork-Presbyterian Hospital, New York, New York

\begin{abstract}
Osteoblastomas are a rare, benign primary bone tumor accounting for $1 \%$ of all primary bone tumors, with $40 \%$ occurring within the spine. Gross-total resection (GTR) is curative, although depending on location, this can require destabilization of the spine and necessitate instrumented fixation. Through the use of minimally invasive, muscle-sparing approaches, these lesions can be resected while maintaining structural integrity of the spine. The authors present a case report and technical note of a single patient describing the use of a purely endoscopic technique to resect a right L5 superior articulating process osteoblastoma in a 45-year-old woman. The patient underwent an image-guided endoscopic resection of her superior articulating facet osteoblastoma. Intraoperative CT demonstrated GTR. On postoperative examination, she remained neurologically intact with resolution of her pain. At follow-up, she remained pain free. Resection of lumbar osteoblastoma through a fully endoscopic approach was a safe and effective technique in this patient. This technique allowed for GTR without compromising spinal structural integrity, thus eliminating the need for instrumented fixation.
\end{abstract}

https://thejns.org/doi/abs/10.3171/2020.2.SPINE191091

KEYWORDS minimally invasive spine surgery; osteoblastoma; stereotactic navigation; spinal endoscopy; spine tumor; oncology; surgical technique

$\mathrm{O}$ STEOBLASTOMAS are rare, benign primary boneforming tumors accounting for $1 \%$ of all primary bone tumors, with $40 \%$ of those in the spine, and can be found within the cortex, medullary canal, or periosteal tissues. ${ }^{1-3}$ Within the spine, osteoblastomas have a predilection for the posterior elements, especially the pedicle and lamina. ${ }^{4}$ Unlike osteoid osteomas, osteoblastomas are larger, more often arise in the axial spine, are not characterized by nocturnal pain, and have a higher rate of recurrence. ${ }^{1}$ Osteoblastomas can have extensive local recurrence after subtotal resection or, rarely, undergo malignant transformation. ${ }^{3}$

When osteoblastomas occur in the spine, resection typically occurs via one of two surgical techniques: curettage intralesional excision, or en bloc resection with the goal of minimizing the risk of recurrence. ${ }^{5,6}$ Recurrence rates vary based on Enneking stage, and recurrence is rare after intralesional excision for contained (stage 2) lesions. ${ }^{7}$ For larger lesions, such en bloc resections necessitate extensive soft-tissue dissection and removal of osseous structures, which potentially results in destabilizing the spine, requiring instrumented fixation as well as an increased surgical morbidity. ${ }^{8}$ To mitigate the risk of destabilization, minimally invasive muscle-sparing approaches can be utilized. ${ }^{9}$ In particular, the use of minimally invasive retractors placed through a $2-\mathrm{cm}$ incision to access these lesions with minimal intraoperative blood loss, short postoperative hospital stays, gross-total resection (GTR) of osseous lesions, and no subsequent need for spinal stabilization has been described.

The use of image-guided, percutaneous endoscopic approaches for the resection of these lesions in the thoracolumbar spine has not been previously reported. This paper describes the GTR of a lumbar osteoblastoma using the percutaneous endoscopic technique and discusses the advantages and limitations of this approach.

\section{Technical Note}

\section{Patient History and Examination}

A 45-year-old woman with no significant past medical history presented to the clinic 2 years prior to surgery with 


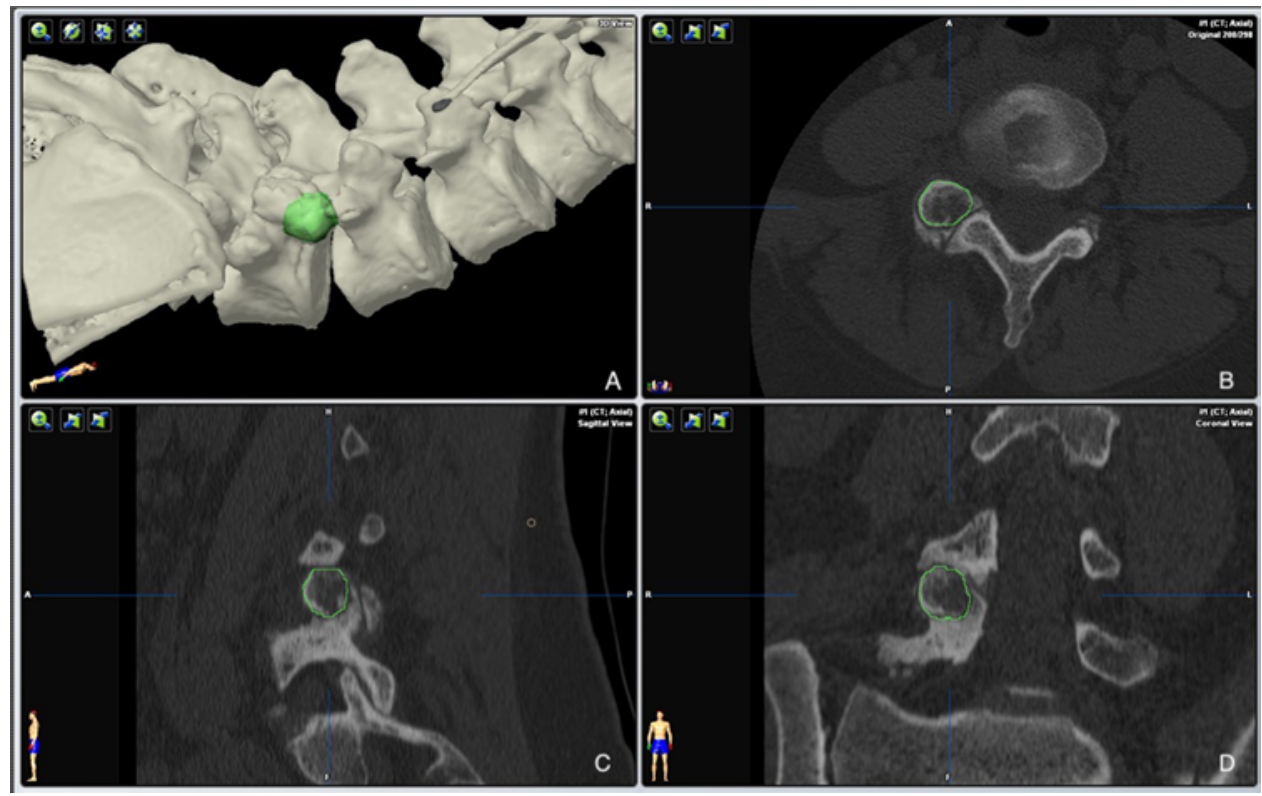

FIG. 1. Preoperative CT image of the lumbar spine without contrast shows 3D reconstruction of the osseous lesion (green) arising from the right L5 superior articulating facet (A). Source images demonstrate the osteolytic lesion (green outline) in axial (B), sagittal (C), and coronal (D) planes. Figure is available in color online only.

concern for an osseous lesion involving the right L5 superior articulating process, most consistent with an Enneking stage 2 osteoblastoma (Fig. 1). She was managed conservatively with observation and antiinflammatory analgesia until she began to suffer worsening right lower-extremity radiculopathy not controlled with pain medications. As discussed with our institutional interventional radiology team, radiofrequency ablation was not pursued as the lesion was deemed to be too close to the exiting right L5 nerve root. The patient was offered an endoscopic partial facetectomy with removal of the L5 superior articulating facet lesion in hopes of avoiding a complete facetectomy that might necessitate a lumbar instrumented fusion. On neurological examination, the patient had full strength in her bilateral lower extremities with normal sensation. She was able to ambulate without difficulty and had a completely benign neurological examination.

\section{Operation}

The patient was positioned prone on the operating frame connected to the Airo Mobile CT Scanner with navigation system (Brainlab). After standard surgical preparation and draping, 2 Steinman pins were inserted into the left iliac crest and connected to the navigation reference array. A helical CT scan of the lumbar spine was obtained for navigation. A subcentimeter incision was made $11 \mathrm{~cm}$ off of midline to the right side in her paraspinal lumbar region. A navigated dilator was used to dock the dilator on the right L5 superior articulating process at the level of the tumor (Fig. 2A and B). An 8-mm beveled working channel was introduced over the dilator and the dilator was replaced with the endoscope (Tessys, Joimax Inc.). To facilitate continuous navigation, a reference array was clamped to the endoscope and registered prior to introduction into the surgical field. A radiofrequency coagulator was used to remove soft tissue overlying the facet joint and visualize the tumor. The tumor was readily visualized under a thin shell of bone distinguished by the light maroon color separating it from the white, sclerotic bone inferior to it (Fig. 3A). A combination of a high-speed diamond drill (Fig. 3B) and a blunt dissector was used to perform an intralesional resection of the tumor. Kerrison rongeurs were used to complete the resection, with care taken to preserve the remainder of the facet joint. Tissue was sent for pathology confirmation. Radiofrequency coagulation (Fig. 3C) was used to meticulously ablate the resection margins and reduce the chances of local recurrence. At this point, an intraoperative CT scan was obtained to assess the extent of tumor removal. CT demonstrated residual osseous tumor inferolaterally, and the endoscope was reintroduced with neuronavigation. After resection of the residual tumor (Fig. 3D), a final helical CT scan was obtained that demonstrated complete resection (Fig. 2C and D) with a competent facet joint. Hemostasis was obtained using radiofrequency coagulation and Floseal (Baxter International), the endoscope was removed, and the wound was irrigated copiously with antibiotic irrigation. The wound was closed with 3-0 Vicryl suture for the deep dermal layer and the skin was closed with a running subcuticular stitch. The resultant incision was approximately $1 \mathrm{~cm}$ in length. Ten milliliters of blood was estimated for the case.

\section{Postoperative Course}

The patient was neurologically intact immediately after surgery with a rapid recovery. She ambulated well 2 hours after extubation and was discharged home less than 24 hours after surgery. At her 6-month follow-up appointment, the patient remained neurologically intact with no 

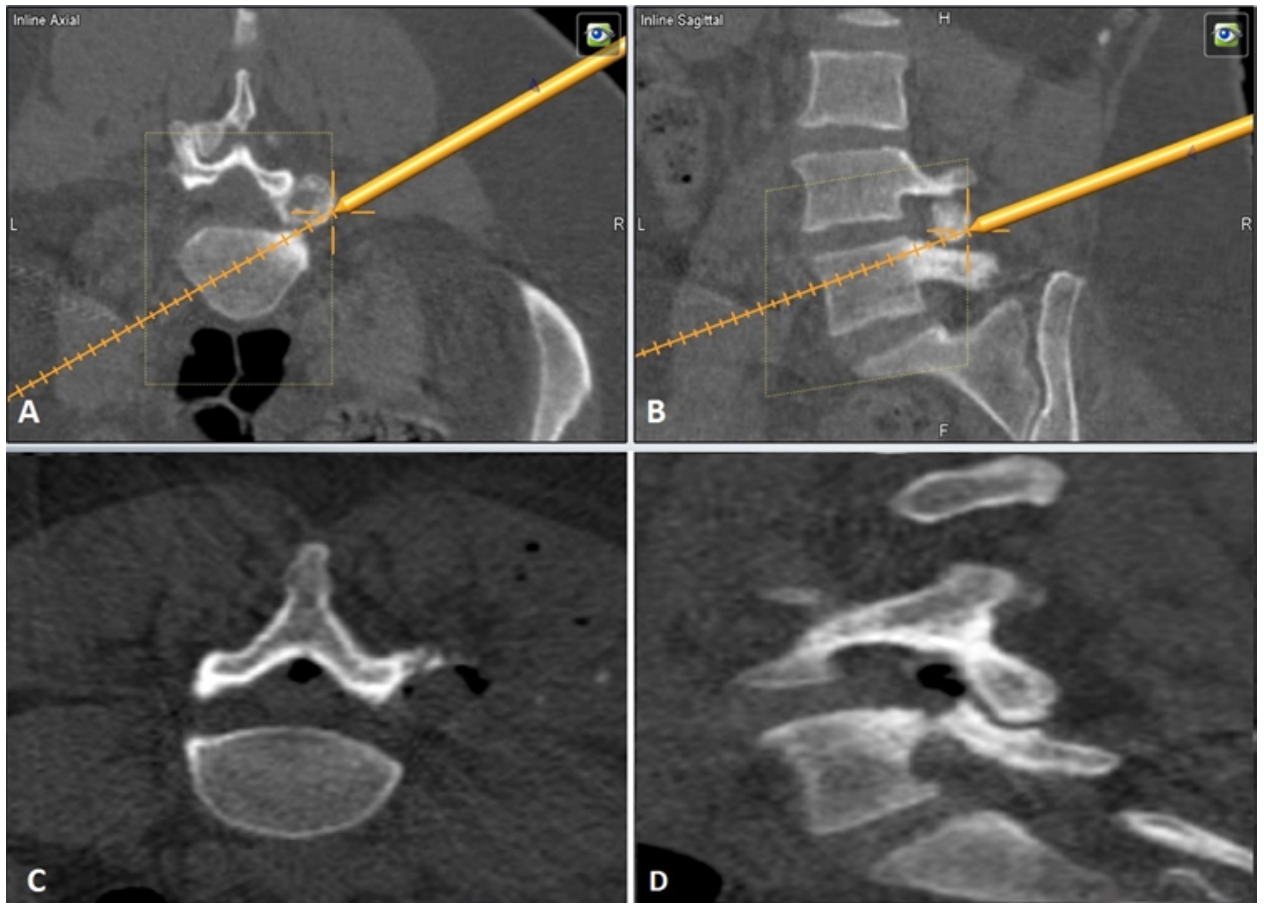

FIG. 2. Initial intraoperative inline axial (A) and sagittal (B) helical CT images with real-time neuronavigation from the endoscope tip (yellow stylet) demonstrate docking on the inferior aspect of the osseous lesion (yellow crosshairs). Final intraoperative CT images with inline axial (C) and sagittal (D) views demonstrate complete resection of the osteoblastoma. Figure is available in color online only.

pain and had resumed physical activity, including running. A pre- and postcontrast MR image of her lumbar spine from the same time demonstrated no signs of residual or recurrent tumor.

\section{Discussion}

The goal of resection of spine osteoblastomas is maximal safe resection, with the goal of GTR to minimize the risk of local recurrence while avoiding neurological deficits. Historically, en bloc resection to achieve wide margins was often advocated, ${ }^{7}$ yet the advantage of wide margins has not been validated in multicenter studies, ${ }^{6}$ prompting the exploration of less invasive surgical strategies. Minimally invasive approaches, conversely, may limit the amount of soft-tissue dissection, intraoperative blood loss, spinal destabilization, and subsequent need for instrumented fixation. ${ }^{4,9}$ However, these minimally invasive approaches predominantly rely upon tubular retractor systems that still require significant dilation and displacement of soft-tissue structures, although with less destruction than standard open approaches.

Radiofrequency ablation has been used as an alternative minimally invasive approach with a smaller incision and less soft-tissue displacement than tubular retractors, but is associated with two important disadvantages.10,11 First, while CT-guided, there is no capability for direct visualization of the radiofrequency probe, which can lead to misplacement and ablation of adjacent neural or paraspinal structures. Second, removal of lesions within the
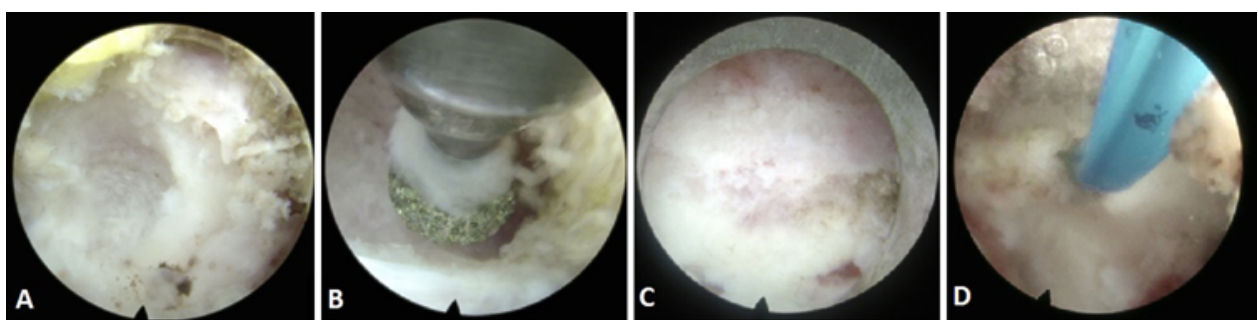

FIG. 3. Image of the osseous lesion after docking, inserting the endoscope, and resecting superficial soft tissues, demonstrating the superficial rim of sclerotic bone overlying the partially drilled tumor $(\mathbf{A})$. Use of the high-speed drill for tumor resection under direct visualization (B). Visualization of the interface of the sclerotic bone (inferior, white-colored) and the tumor tissue (C; superior, mauve-colored). Radiofrequency coagulation of the tumor margins for hemostasis and reduction of the risk of tumor recurrence (D). Figure is available in color online only. 
spinal canal or adjacent to neural foramina (as in this case) is not possible due to the risk of injuring nearby neural structures such as exiting nerve roots.

In this paper, we describe the first reported case of the use of an image-guided, percutaneous, fully endoscopic approach for resection of a spinal osseous tumor. This approach combines the benefits of direct visualization with the smaller incision and reduced muscle disruption noted with percutaneous radiofrequency ablation techniques. Additionally, the endoscopic technique allows for the resection of lesions adjacent to neural structures with direct visualization. In our case, we were able to obtain GTR with no consequent neurological deficits, minimal blood loss, and no need for intravenous postoperative pain medications.

While this technique brings with it the aforementioned advantages, there are also several limitations that must be mentioned. First, the direct visualization gained by using working channel endoscopy can be obscured by tumoral bleeding that may be difficult to stop; therefore, this approach is not amenable to the resection of highly vascular tumors. Second, in patients with large tumors in whom complete resection of the tumor would destabilize the spine, this approach does not allow for simultaneous instrumentation and would necessitate a combined percutaneous fusion procedure. Third, while the tumor can be directly visualized, technical considerations can predispose the surgeon to incomplete resection of the tumor. This predisposition can be overcome with the use of intraoperative CT to confirm the extent of resection.

Overall, we describe a novel application of percutaneous spinal endoscopy for the resection of spinal osseous tumors, and our case demonstrates the feasibility, safety, and efficacy of this image-guided approach.

\section{References}

1. Atesok KI, Alman BA, Schemitsch EH, et al. Osteoid osteoma and osteoblastoma. J Am Acad Orthop Surg. 2011;19(11):678-689.

2. Limaiem F, Singh R. Cancer, Osteoblastoma. In: StatPearls. StatPearls Publishing; 2019. http://www.ncbi.nlm.nih.gov/ books/NBK536954/. Accessed February 18, 2020.
3. Lucas DR, Unni KK, McLeod RA, et al. Osteoblastoma: clinicopathologic study of 306 cases. Hum Pathol. 1994;25(2):117-134.

4. Haji FA, Cenic A, Crevier L, et al. Minimally invasive approach for the resection of spinal neoplasm. Spine (Phila Pa 1976). 2011;36(15):E1018-E1026.

5. Berry M, Mankin H, Gebhardt M, et al. Osteoblastoma: a 30-year study of 99 cases. J Surg Oncol. 2008;98(3):179-183.

6. Versteeg AL, Dea N, Boriani S, et al. Surgical management of spinal osteoblastomas. J Neurosurg Spine. 2017;27(3):321327.

7. Boriani S, Amendola L, Bandiera S, et al. Staging and treatment of osteoblastoma in the mobile spine: a review of 51 cases. Eur Spine J. 2012;21(10):2003-2010.

8. Galgano MA, Goulart CR, Iwenofu H, et al. Osteoblastomas of the spine: a comprehensive review. Neurosurg Focus. 2016;41(2):E4.

9. Regev GJ, Salame K, Keynan O, Lidar Z. Resection of benign vertebral tumors by minimally invasive techniques. Spine J. 2015;15(11):2396-2403.

10. Martel J, Bueno A, Nieto-Morales ML, Ortiz EJ. Osteoid osteoma of the spine: CT-guided monopolar radiofrequency ablation. Eur J Radiol. 2009;71(3):564-569.

11. Osti OL, Sebben R. High-frequency radio-wave ablation of osteoid osteoma in the lumbar spine. Eur Spine J. 1998;7(5):422-425.

\section{Disclosures}

Dr. Laufer reports being a consultant to Medtronic, Globus, DePuy Synthes, Brainlab, and SpineWave.

\section{Author Contributions}

Conception and design: Barzilai. Acquisition of data: Barzilai, Newman. Analysis and interpretation of data: Barzilai, Newman. Drafting the article: all authors. Critically revising the article: all authors. Reviewed submitted version of manuscript: all authors. Approved the final version of the manuscript on behalf of all authors: Barzilai. Study supervision: Barzilai.

\section{Correspondence}

Ori Barzilai: Memorial Sloan Kettering Cancer Center, New York, NY.barzilao@mskcc.org. 